\title{
The many faces of gallbladder cancer: a pictorial review
}

Andreia Tereso, Ana Germano, Marta Baptista, Ana Nassauer Mónica

Hospital Prof Doutor Fernando Fonseca, EPE, Amadora, Portugal

\section{Learning objectives}

- To alert to the importance of gallbladder cancer.

- To describe the clinical and imaging findings of gallbladder cancer based on a review of pathologically confirmed cases diagnosed in our institution.

Epidemiology: fifth most common gastrointestinal cancer; the most common type of biliary cancer; median age of presentation is 72 years; diagnosis is more common in women and in the elderly.
Clinical presentation: early symptoms of gallbladder cancer overlap with those attributed to gallstone disease; unspecific symptomatology (chronic abdominal pain, weight loss, and anorexia) leads to diagnosis at advanced inoperable stages.

Risk factors: demographic - female gender, postmenopausal status, age, and increased body mass; environmental - cigarette smoking, and exposure to chemicals in rubber, automobile, wood finishing, and metal fabricating industries; chronic infections (Salmonella tiphy); inflammatory status: porcelain gallbladder primary sclerosing cholangitis and cholelithiasis congenital conditions, like low insertion of the cystic duct
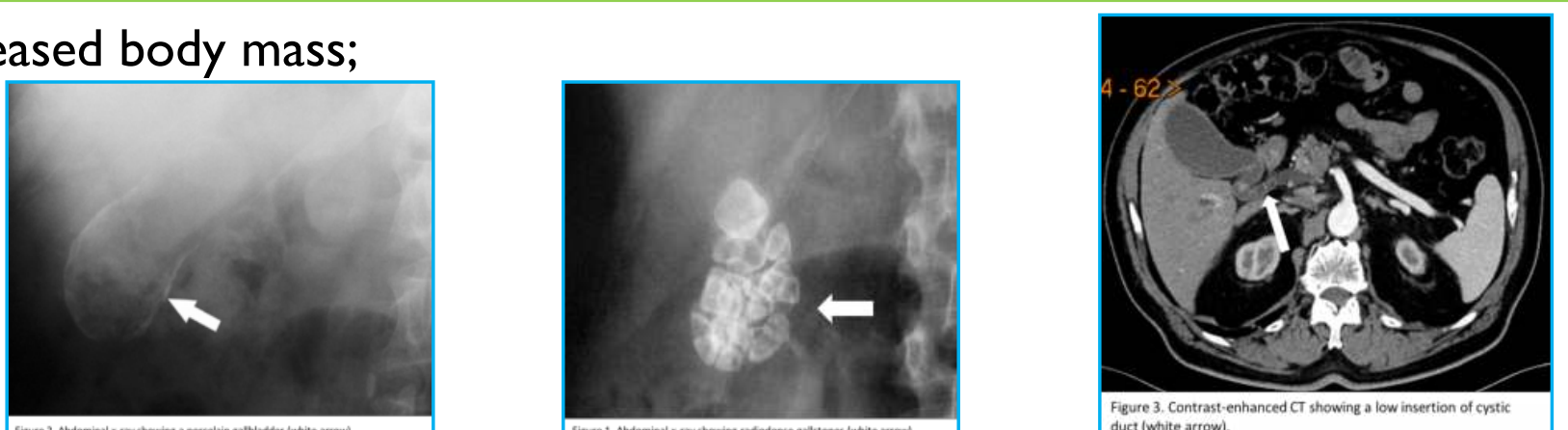

Pathological aspects: the lack of muscularis mucosae and subserosa layers eases the involvement of adjacent organs by the gallbladder carcinoma; based on American Joint Committee on Cancer $(\mathrm{AJCC})$ the gallbladder cancer can be staged according to the TNM system.

Surgical treatment: early stages may benefit from extended ressection after cholecystectomy; in advanced stages, radical surgery is contorversial.

Ultrasound features of gallbladder carcinoma: gallstones with absence of other sonographic findings

$\checkmark$ an intraluminal polypoid lesion

$\checkmark$ a focal or diffuse asymmetric parietal thickening

acute or chronic cholecystitis
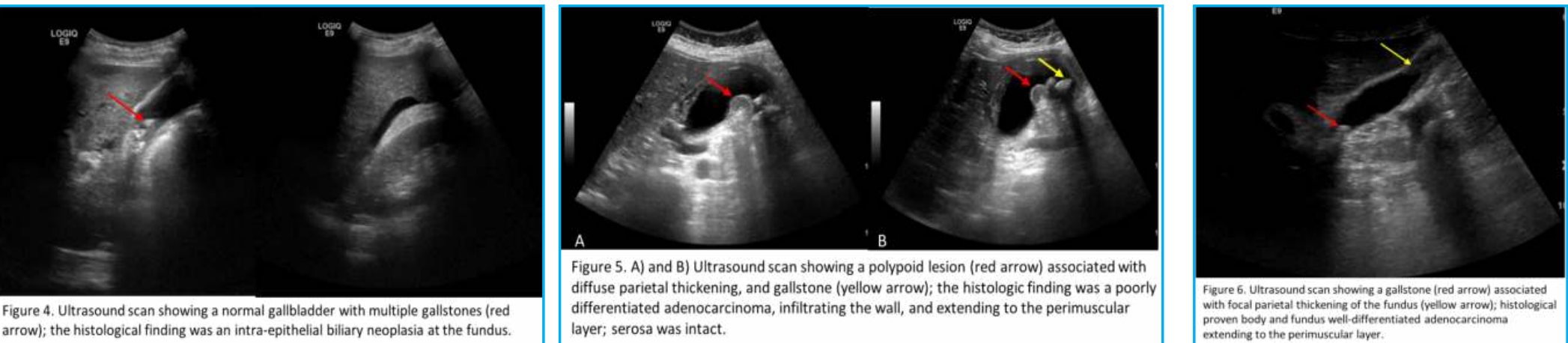

$\checkmark$ gallstones associated with a gallbladder mass

$\checkmark$ intra-hepatic bile ducts dilatation and heterogeneous mass filing the gallbladder lumen and invades the adjacent liver/duodenum
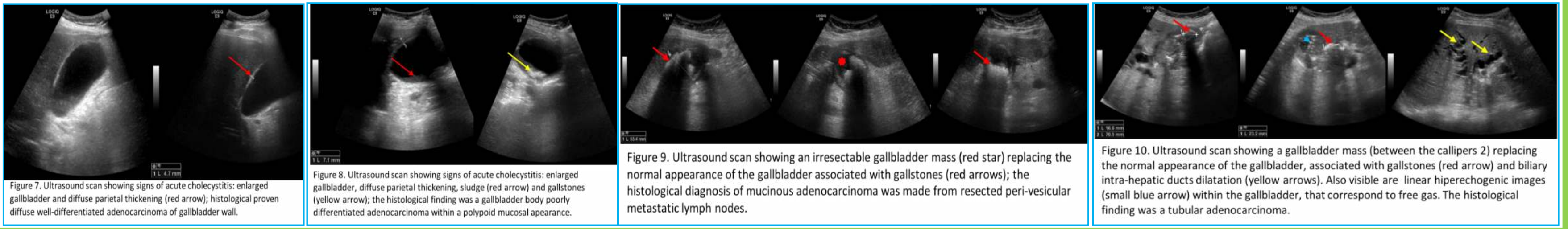

CT features of gallbladder carcinoma:

$\checkmark$ an intraluminal polypoid lesion

$\checkmark$ an asymmetric, irregular wall thickening that enhances during the arterial phase

$\checkmark$ a heterogeneous mass within the gallbladder fossa

$\checkmark$ a hepatic abscess

$\checkmark$ a mass that invades the adjacent liver parenchyma or the duodenum

$\checkmark$ diffuse liver metastatic disease
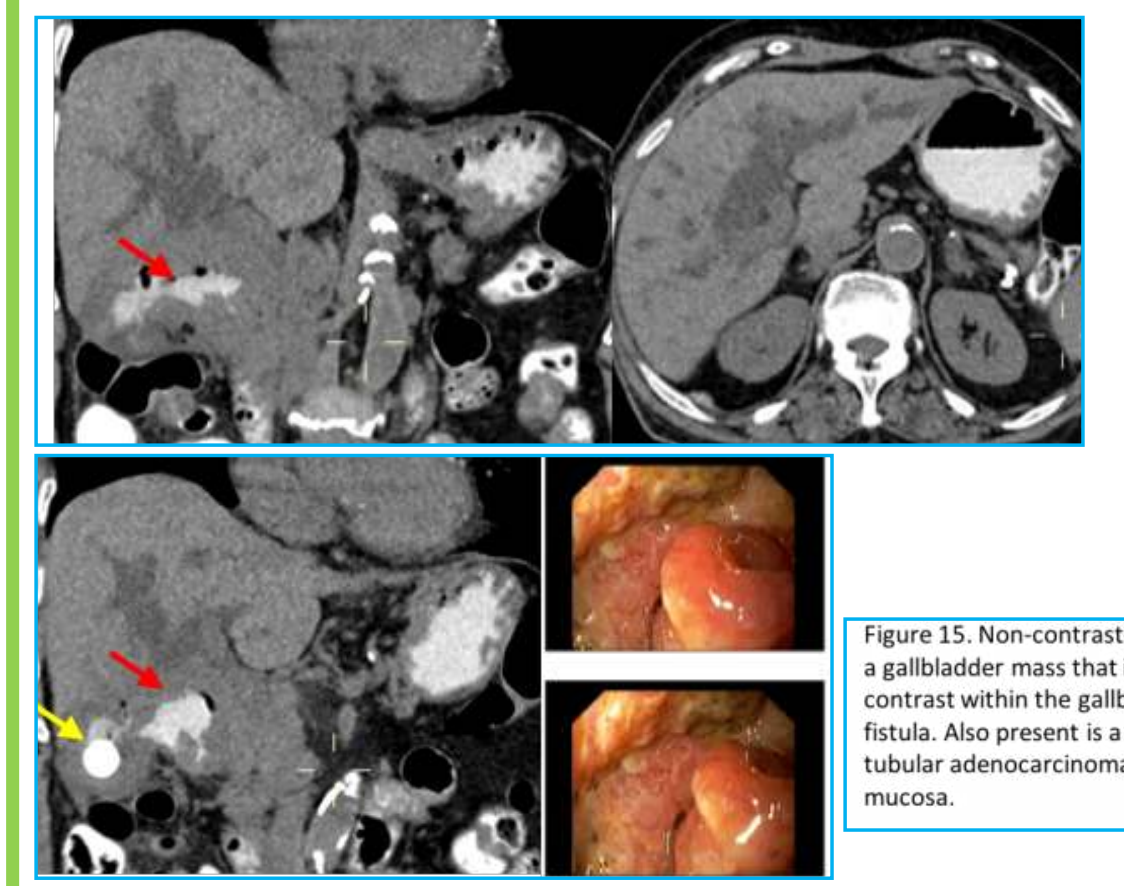
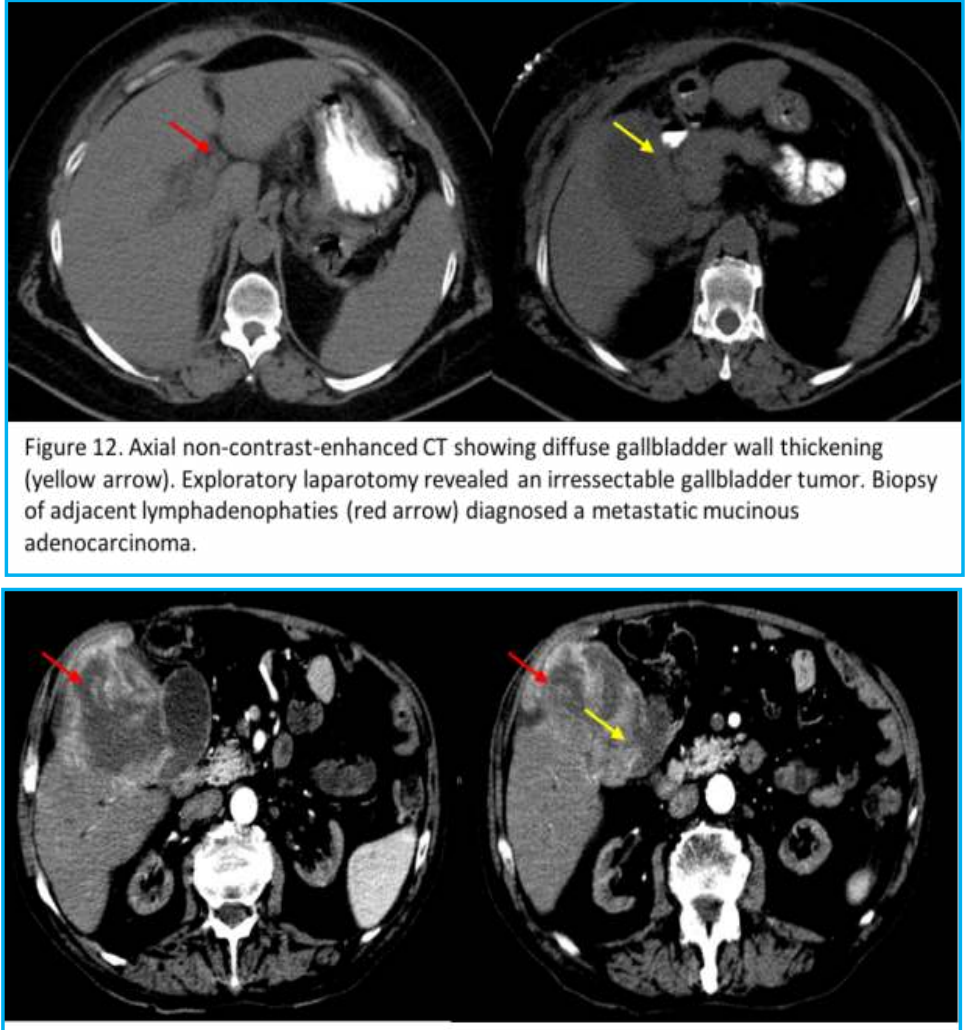

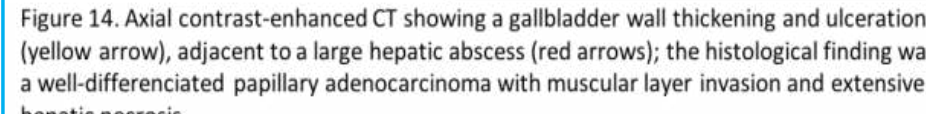
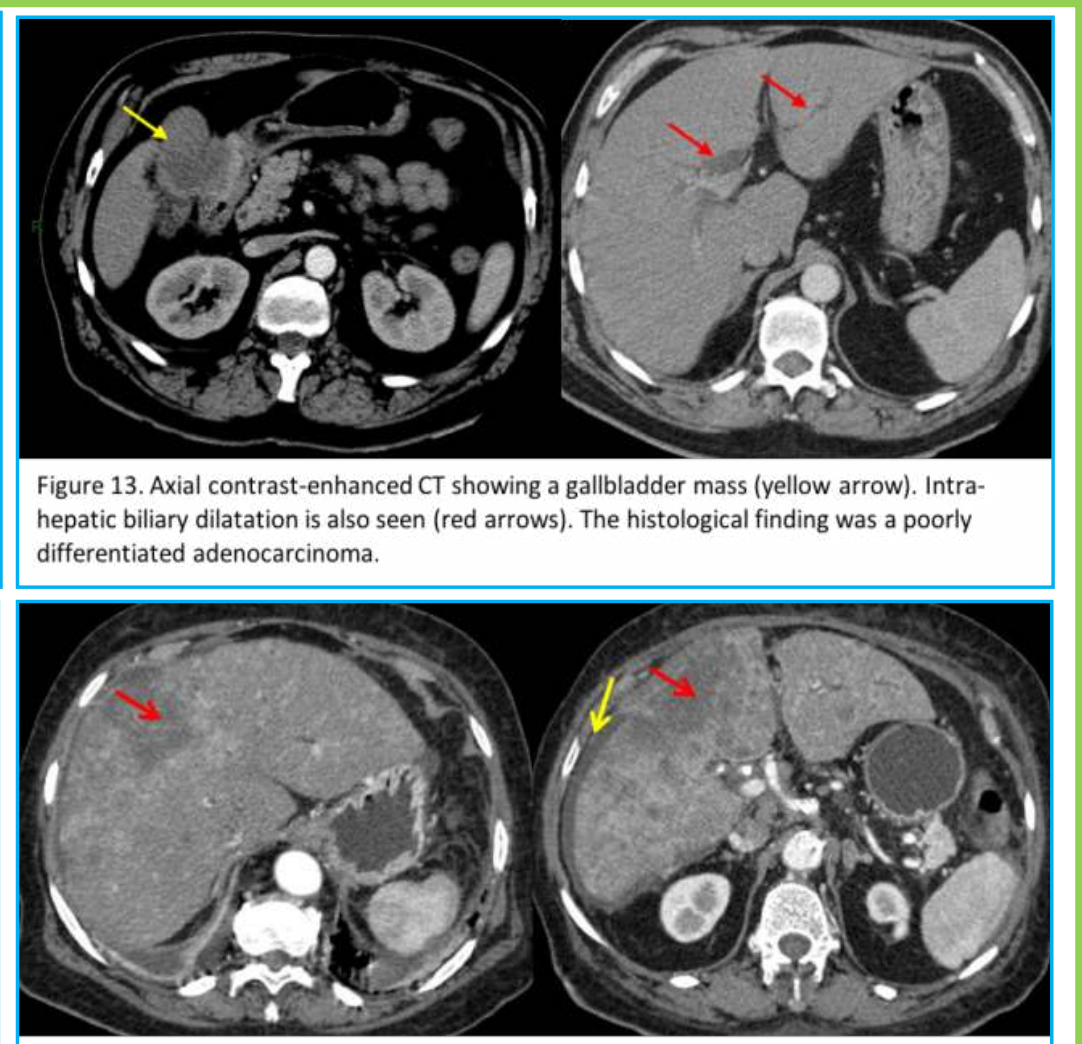
Figure 16. Contrast-enhanced computed tomography showing multiple hepatic met
(red arrows). Ascites is also seen (yellow arrow). The histological finding after
cholecystectomy revealed a mucinous differentiated gallbladder adenocarcinoma
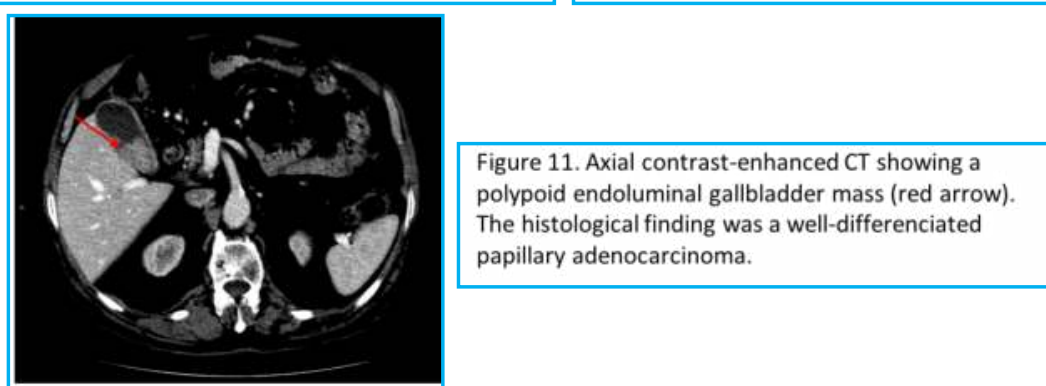

Our series: $53 \%$ of our patients were women; median age at presentation was 66,2 years; $73 \%$ presented in the emergency department with an acute onset of symptoms; in $2 / 3$ of our patients, gallbladder carcinoma was an incidental histological finding after cholecystectomy; all patients underwent a CT scan for staging; six patients were submitted to an extended resection.

Conclusion: The awareness of "the many faces of gallbladder cancer" is crucial to promptly recognise suspicious signs of this pathology to provide an early diagnosis and to prevent the high mortality related to this type of cancer. 